Recherches en didactique des langues et des cultures

Les cahiers de l'Acedle

13-3 | 2016

Travailler sur et à partir de textes écrits en classe de langue étrangère

\title{
L'analyse des obstacles comme source de dépassement des difficultés en production écrite
}

Joaquim Dolz, Roxane Gagnon et Yann Vuillet

\section{(2) OpenEdition}

Journals

Édition électronique

URL : http://journals.openedition.org/rdlc/1234

DOI : $10.4000 /$ rdlc. 1234

ISSN : 1958-5772

Éditeur

ACEDLE

Référence électronique

Joaquim Dolz, Roxane Gagnon et Yann Vuillet, «L'analyse des obstacles comme source de dépassement des difficultés en production écrite », Recherches en didactique des langues et des cultures [En ligne], 13-3 | 2016, mis en ligne le 31 décembre 2016, consulté le 19 avril 2019. URL http://journals.openedition.org/rdlc/1234; DOI : 10.4000/rdlc.1234

Ce document a été généré automatiquement le 19 avril 2019

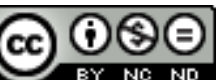

Recherches en didactique des langues et des cultures is licensed under a Creative Commons AttributionNonCommercial-NoDerivatives 4.0 International License 


\title{
L'analyse des obstacles comme source de dépassement des difficultés en production écrite
}

\author{
Joaquim Dolz, Roxane Gagnon et Yann Vuillet
}

\section{Introduction ${ }^{1}$}

1 L'objet de cette contribution est de présenter une démarche explicitement orientée vers l'analyse des productions écrites, laquelle permet de dégager les capacités et les difficultés des élèves auteurs de ces textes (Dolz, Gagnon \& Vuillet, 2011). Les supports didactiques permettant la représentation de la situation de communication lors de l'écriture sont analysés au travers de productions écrites d'élèves du primaire et d'étudiants de français langue étrangère. L'apport principal de cette approche consiste à prendre appui sur les capacités et les erreurs des élèves pour organiser l'enseignement. L'analyse des erreurs nous permet de voir les obstacles auxquels les élèves se heurtent dans l'apprentissage de la production écrite. De même, l'analyse permet d'opérationnaliser le concept de zone de développement proche (Vygostki, 1934/1997). Cette opérationnalisation permet également de penser les dispositifs d'enseignement et des formes d'interventions différenciées en fonction des apprenants de chaque groupe (différentiation interne) et des besoins d'adaptation aux deux groupes contrastés (différentiation externe) : enfants vs adultes et français langue première vs français langue étrangère

2 Pour travailler la production textuelle, les tâches d'écriture présentées par les enseignants peuvent comporter divers supports (feuilles volantes, fiches d'exercice, textes lacunaires, documents à l'appui, matrice textuelle, etc.). Cet ensemble de supports constitue un dispositif didactique servant, d'une part, à la représentation de la situation de communication du genre de texte à écrire et, d'autre part, au pointage des contenus thématiques et des dimensions linguistiques à travailler. Ici, nous nous intéressons aux 
supports dans la rédaction de deux genres argumentatifs: la lettre de demande (désormais LD) et la réponse au courrier des lecteurs (RCL). Il s'agit de feuilles volantes, lesquelles incluent l'énoncé de la consigne et l'espace du texte à écrire. Ces supports donnent lieu à des productions d'élèves dont l'analyse permet de procéder à un enseignement plus ciblé.

Les notions de texte et de genre textuel sont à la base de l'analyse des obstacles rencontrés par les apprenants. L'analyse du fonctionnement discursif des textes sociaux de référence et les textes produits par les élèves permet de les analyser comme des systèmes sémiotiques (Bronckart, 1997). Tout texte produit par un apprenant est considéré comme un exemplaire particulier d'un genre de texte, et chaque genre textuel est abordé par les enseignants comme objet d'enseignement. La généricité des textes produits est étudiée dans le cadre des situations d'interaction et dans l'espace des contraintes qui interviennent dans le fonctionnement discursif. Un ensemble de critères linguistiques et extralinguistiques sont pris en considération pour y parvenir. Les observables textuels sont à la base de la description des phénomènes explorés : aussi bien ceux qui relèvent de la cohérence générique ou de la configuration linguistique spécifique que ceux pris comme révélateurs d'un dysfonctionnement ou d'un apprentissage en cours de construction (Dolz \& Abouzaid, 2015).

4 La modélisation didactique des deux genres scolarisés étudiés permet une clarification des finalités d'apprentissage relatives à la communication ciblées par les enseignants et articulées aux moyens linguistiques qui la rendent possible. L'optique contrastive proposée entre deux genres argumentatifs, lettre de demande et lettre au courrier des lecteurs, contribue à identifier les régularités et les spécificités génériques. Les modèles didactiques des genres dans une approche contrastive, en plus de mettre en évidence ce qui peut présenter de l'intérêt pour l'enseignement, fournissent une grille des critères et des indicateurs pour évaluer les capacités et les difficultés des apprenants. Nous proposons ainsi une démarche pour identifier les principaux obstacles des apprenants (aussi bien adultes de langue étrangère qu'élèves de l'école primaire), tandis que la caractérisation de chaque genre fournit un inventaire de lieux d'observation pour saisir les tensions entre enseignement et apprentissage: cet inventaire nous apparait fondamental pour l'évaluation de la production écrite.

5 Afin d'expliciter notre démarche, nous procédons d'abord par une présentation du corpus analysé. Puis, nous présentons les modèles didactiques des genres qui servent de base à l'analyse des obstacles contenus dans les deux corpus. Nous procédons ensuite à l'analyse contrastée des erreurs et des capacités des apprenants afin de dégager les principaux obstacles et les zones de développement (Vygotski, 1934/1997). Les difficultés d'écriture des apprenants se manifestent surtout par les erreurs et les dysfonctionnements dans les textes. Le statut des dysfonctionnents constatés seront mis en relation avec les obstacles que l'apprenant doit dépasser pour rédiger son texte. La catégorisation des obstacles est forcément hétérogène mais s'organise en fonction des savoirs mobilisés à propos des genres argumentatifs étudiés et sur la langue française (savoirs et savoir-faire lexicaux, syntaxiques et orthographiques, par exemple). Elle prend aussi en considération les opérations sous-jacentes à la production textuelle (contextualisation, planification, textualisation et révision, principalement) ainsi que les mécanismes d'apprentissage en fonction de l'âge des apprenants (adultes vs enfants). Elle prend également en considération le statut de la langue (première ou étrangère) et du niveau de connaissances initiales. Enfin, des pistes de dispositifs d'enseignement adaptés et 
différenciés permettant de dépasser les obstacles évoqués seront proposées. L'ambition de l'approche comparatiste consiste à explorer les spécificités et les points de convergence entre les deux publics d'apprenants contrastés.

\section{Deux corpus de textes issus de situations et de pratiques d'enseignement contrastées}

Les deux corpus de textes sont issus de pratiques d'enseignement observées par les chercheurs. Les contextes de travail, les tâches proposées, les genres argumentatifs choisis et les démarches de travail sont différentes. Le premier corpus se compose de productions de textes argumentatifs du genre « lettre au courrier de lecteur » d'élèves de $6^{e}$ année primaire (9-10 ans) recueillis dans des classes du canton du Valais qui travaillent dans une démarche en séquences didactiques (Dolz, Noverraz \& Schneuwly, 2001). Le support pour l'écriture est une feuille volante incluant la consigne de production et la lettre de lecteur à laquelle l'élève doit répondre. Le second corpus rassemble des productions d'une lettre de demande d'étudiants adultes d'une classe de français langue étrangère (niveau B2-C1) de l'École de Civilisation et de Langue Française (désormais ECLF). Les supports sont constitués d'une feuille volante avec énoncé de consigne et espace blanc à remplir dans le cas du corpus.

\section{Corpus d'étudiants FLE adultes}

7 Le corpus «ELCF » provient d'une unité de formation intitulée Atelier d'écriture. Il s'agit d'étudiants poursuivant un Diplôme d'études de français langue étrangère à la Faculté des Lettres de l'université de Genève, à l'École de langue et de civilisation françaises, au cours de l'année universitaire 2011-2012. Parmi les langues d'origine des étudiants, on retrouve le persan (farsi), l'hébreu, le russe, le bulgare, l'albanien, l'ukrainien, le mongole, le chinois, le japonais, l'anglais, l'allemand et le tagalog. Les objectifs de l'atelier sont les suivants: "assurer les savoirs et savoir-faire du niveau B2 du Cadre Européen de Référence et exercer ceux du niveau $\mathrm{C} 1 \%$. Ce qui signifie que les étudiants doivent pouvoir écrire des textes clairs et détaillés, de divers genres, en développant un point de vue et en faisant la synthèse d'informations empruntées à des sources diverses. La diversité des textes doit servir à l'entrainement de structures linguistiques essentielles.

\section{Corpus d'élèves en français langue première}

Le corpus « Valais 6e primaire » représente une fraction d'un corpus plus vaste, qui visait à contraster les difficultés rencontrées par des élèves de fin de sixième année qualifiés de «faibles » par leurs enseignants en expression écrite et celles éprouvées par des élèves perçus comme « forts » dans le même domaine.

Plus précisément, cette recherche a décrit les capacités et les difficultés des apprenants en situation de production d'un genre textuel à visée argumentative - la réponse au courrier des lecteurs (RCL) - (Dolz, Vuillet, Mabillard, Tobola, 2009). Elle a aussi permis de décrire et d'analyser certaines conditions d'enseignement et d'apprentissage d'un tel genre argumentatif en prenant en considération, pour une analyse de cas, les interactions 
entre l'enseignant et les élèves lors du déroulement d'une séquence d'enseignement ( Vuillet, Y., Mabillard, J.-P., Tobola-Couchepin, C. \& Dolz, J., 2012).

Pour le corpus «Valais 6e primaire» qui nous concerne ici, nous avons retenu 16 productions d'élèves, signalés comme étant en difficulté par leurs enseignants respectifs, et issus de 6 classes différentes. Toutes ces RCL ont été produites au début d'une séquence d'enseignement reposant sur l'utilisation des moyens d'enseignement officiels suisses romands « S'exprimer en français » (2001): dans l'esprit de ces moyens, les productions initiales permettent aux enseignants de collecter des informations quant aux connaissances préalables et aux représentations que les élèves se font du genre de texte $\mathrm{RCL}$, et d'adapter en conséquence leurs enseignements.

\section{Modélisations didactiques des deux genres argumentatifs étudiés}

11 Les genres argumentatifs abordés présentent des caractéristiques génériques qui peuvent être contrastées à différents niveaux. La modélisation didactique des genres (de Pietro J.F., Erard, S. Kaneman 1997 ; Dolz \& Schneuwly, 1998) permet d'identifier non seulement les caractéristiques de la base d'orientation des deux situations de communication, mais également les traits génériques de l'organisation de leur planification textuelle ainsi que, au niveau de la mise en texte, les principales unités linguistiques saillantes. En situation scolaire, les deux genres ont été didactisés par les enseignants comme outils sociaux de communication (avec une visée d'intervention sur l'opinion d'un destinataire dans un lieu social déterminé). Dans ce cadre, leurs principales dimensions ont été transformées en objets d'apprentissage scolaire en français (Schneuwly \& Dolz, 1997).

Au-delà de l'intérêt des modèles didactiques pour générer des séquences d'enseignement, ils constituent une aide pour formuler des hypothèses à propos des apprentissages attendus ou exprimés par les élèves (Dolz, Gagnon \& Vuillet, 2011). Les deux modèles didactiques que nous présentons ci-dessous en forme de schémas synthétiques permettent de pointer une sorte de «forme idéale » du savoir-faire visé qui contribue à déterminer les capacités langagières révélées ainsi que les principaux obstacles des apprenants. Et ceci aux trois niveaux retenus pour étudier les éventuelles difficultés dans la production d'un texte :

1. la base d'orientation aide à vérifier la capacité des élèves à s'adapter aux situations de communication ;

2. les régularités de l'organisation du genre permettent d'en analyser la planification;

3. les éléments de la textualité qui caractérisent le fonctionnement linguistique des genres peuvent aider, non seulement à détecter les connaissances, les capacités et les obstacles linguistiques, mais aussi à clarifier les opérations et les procédés de mise en texte en cours d'apprentissage.

\section{Modèle didactique du genre « lettre de demande »}

\begin{tabular}{|l|l|}
\hline \multicolumn{2}{|l|}{ Base d'orientation } \\
\hline But(s) & $\begin{array}{l}\text { Prendre position par rapport à une controverse en justifiant son } \\
\text { point de vue par un ensemble d'arguments }\end{array}$ \\
\hline
\end{tabular}




\begin{tabular}{|c|c|}
\hline \multirow{3}{*}{$\begin{array}{l}\text { Paramètres du contexte } \\
\text { de communication }\end{array}$} & Lieu social : la correspondance formelle \\
\hline & Énonciateur/argumentateur : l'auteur de la lettre \\
\hline & Destinataire : celui ou ceux à qui la demande est formulée \\
\hline Contenus thématiques & Les contenus thématiques autour des demandes \\
\hline \multicolumn{2}{|l|}{ Planification } \\
\hline \multirow{3}{*}{ Macro-planification } & $\begin{array}{l}\text { Mise en page: présence de la date et du lieu; nom et adresse du } \\
\text { destinataire; formule de salutation, corps de la lettre, formules de } \\
\text { clôture et de salutations finales, signature. }\end{array}$ \\
\hline & $\begin{array}{l}\text { Parties du corps de la lettre: contextualisation de la demande, } \\
\text { demande et justification }\end{array}$ \\
\hline & Ordre logique des parties de la lettre \\
\hline Micro-planification & $\begin{array}{l}\text { Cohérence interne de chacun des paragraphes : progression thème/ } \\
\text { rhème (lois de progression, répétition, non-contradiction) }\end{array}$ \\
\hline \multicolumn{2}{|l|}{ Textualisation } \\
\hline Cohésion verbale & $\begin{array}{l}\text { Base temporelle au présent maintenue; les autres temps de verbes } \\
\text { utilisés sont appropriés }\end{array}$ \\
\hline Cohésion nominale & Phénomènes de reprise \\
\hline $\begin{array}{l}\text { Mécanismes de } \\
\text { connexion }\end{array}$ & Organisateurs logico-argumentatifs. \\
\hline Lexique/vocabulaire & $\begin{array}{l}\text { Selon le ton adopté par l'argumentateur; formules pour marquer son } \\
\text { accord ou son désaccord }\end{array}$ \\
\hline $\begin{array}{l}\text { Marqueurs de } \\
\text { modalisation }\end{array}$ & $\begin{array}{l}\text { Marques de nuances, utilisation du conditionnel, du subjonctif, des } \\
\text { auxiliaires de modalité, des adjectifs et des adverbes. }\end{array}$ \\
\hline $\begin{array}{l}\text { Marqueurs de prise en } \\
\text { charge énonciative }\end{array}$ & $\begin{array}{l}\text { Régie des différentes instances énonciatives : emploi des déictiques et } \\
\text { des pronoms }\end{array}$ \\
\hline
\end{tabular}

\section{Modèle didactique du genre « réponse au courrier des lecteurs »}

\begin{tabular}{|c|c|}
\hline \multicolumn{2}{|l|}{ Base d'orientation } \\
\hline But(s) & Donner son avis quant à la controverse de la lettre source. \\
\hline $\begin{array}{l}\text { Paramètres du contexte } \\
\text { de communication }\end{array}$ & $\begin{array}{l}\text { Lieu social: la rubrique "réponse au courrier des lecteurs " d'une } \\
\text { publication pour enfants. }\end{array}$ \\
\hline
\end{tabular}




\begin{tabular}{|c|c|}
\hline & $\begin{array}{l}\text { Énonciateur : le lecteur de la lettre initiale, l'énonciateur et l'auteur } \\
\text { coïncident. }\end{array}$ \\
\hline & $\begin{array}{l}\text { Destinataires: L'auteur de la lettre d'origine, les acteurs de la } \\
\text { controverse évoquée, le responsable de la rubrique RCL, les lecteurs } \\
\text { du journal (peuvent ne pas avoir lu la lettre source). }\end{array}$ \\
\hline \multirow{4}{*}{ Contenus thématiques } & Synthèse de la controverse de la lettre source. \\
\hline & Opinion personnelle. \\
\hline & Phases d'argumentation. \\
\hline & $\begin{array}{l}\text { Les contenus référentiels sont plausibles et cohérents. Ils ont un } \\
\text { degré élevé de généralité (pour l'efficacité de l'argumentation). }\end{array}$ \\
\hline \multicolumn{2}{|l|}{ Planification } \\
\hline \multirow{5}{*}{ Macro-planification } & $\begin{array}{l}\text { Titre en rapport avec la controverse ou cohérent avec l'avis défendu } \\
\text { (facultatif). }\end{array}$ \\
\hline & $\begin{array}{l}\text { Organisation des contenus en fonction de deux logiques: informative } \\
\text { et argumentative. }\end{array}$ \\
\hline & $\begin{array}{l}\text { Partie argumentative: contextualisation de la controverse, } \\
\text { développement et hiérarchisation des arguments, conclusion. }\end{array}$ \\
\hline & Paragraphes en fonction des contenus. \\
\hline & Formules épistolaires d'introduction et de conclusion. \\
\hline Micro-planification & $\begin{array}{l}\text { Progression thème/rhème (lois de progression, répétition, non- } \\
\text { contradiction). }\end{array}$ \\
\hline \multicolumn{2}{|l|}{ Textualisation } \\
\hline Cohésion verbale & Base au présent de l'indicatif. \\
\hline Cohésion nominale & Reprises anaphoriques/cataphoriques. \\
\hline $\begin{array}{l}\text { Mécanismes de } \\
\text { connexion }\end{array}$ & Organisateurs spatiotemporels; organisateurs logico-argumentatifs. \\
\hline \multirow{2}{*}{$\begin{array}{l}\text { Mécanismes de prise en } \\
\text { charge énonciative }\end{array}$} & $\begin{array}{l}\text { Unités déictiques et modalisations personnelles dans la partie } \\
\text { argumentative. }\end{array}$ \\
\hline & Marques impersonnelles dans la partie informative. \\
\hline
\end{tabular}

Les items présentés dans les modélisations génériques correspondent aux éléments et aux contours caractéristiques des genres qui peuvent faire l'objet d'activités d'observation et d'enseignement de la production écrite. Ici, nous les prendrons en référence pour 
l'évaluation des productions des élèves, sans pouvoir entrer dans le détail pour les indicateurs et les critères retenus.

En fonction des trois niveaux d'analyse retenus, les questions qui ont orienté les analyses sont les suivantes :

- Dans les supports proposés par les enseignants, quels sont les éléments retenus par les apprenants pour adapter le texte à la situation de communication ?

- Comment les supports contribuent-ils aux choix des arguments et à la planification du texte?

- À partir de ces supports, quels sont les principaux obstacles des élèves au moment de la mise en texte du genre argumentatif?

\section{Analyse du corpus de lettres d'étudiants de FLE adultes}

\section{Premier niveau : sur la situation de communication}

La consigne de production du corpus «ECLF » apparait sur la feuille support que les étudiants doivent remplir. La situation de communication proposée par cette consigne se veut réaliste: "Vous aviez l'intention de suivre le parcours A, avec le renforcement linguistique, mais vous venez d'apprendre que la direction de l'ECLF a décidé de le supprimer pour des raisons budgétaires ». Les étudiants doivent donc écrire une lettre au directeur de l'institution, pour lui demander de revenir sur cette décision. Notons que certains contenus de la lettre sont attendus, la consigne leur demandant d'expliquer brièvement leurs motivations et leurs besoins.

Dans l'ensemble, la consigne a été comprise par les étudiants. Ceux-ci ont rédigé une lettre de demande, écrite à la première personne, adressée au directeur. Les étudiants parlent en leur nom, mais aussi au nom de tous les étudiants allophones à qui un tel programme est nécessaire. Ceci se voit dans le jeu des pronoms utilisés dans les productions :

(1) * «je voudrais vous dire que c'est un cours qu'on besoin pour nous améliorer et totalement nécessaire si on veut avoir un renforcement linguistique ",

(2) * «J'espère que nos petits lettres peuvent changer votre décision ».

Les étudiants invitent le professeur Gajo à revenir sur sa décision et à ne pas supprimer le parcours A et ce, en dépit des restrictions budgétaires. Certains étudiants sollicitent un échange ou une rencontre avec Gajo en guise de prolongement de la demande :

$(3){ }^{*}$ « je reste à votre disposition pour elaboré sur ces thème en dessous ».

Les quelques copies où l'on note des difficultés en lien avec la représentation de la situation de communication ne sont pas marquées par des erreurs dans la prise en charge énonciative, mais se rattachent à la représentation générale des paramètres du contexte de production ou, à ce qui relève de la fictionnalisation de la situation de communication (Schneuwly, 1997). À une occasion la demande est absente ; à une autre, elle est formulée en termes vagues « Pourriez-vous réfléchir encore une fois sur ce sujet?». Deux autres l'interprètent de la manière suivante: c'est parce qu'ils ont une situation budgétaire 
précaire qu'on les prive du renforcement linguistique. La demande au directeur prend alors de tournures plus personnelles :

(4) * «Mais je vous jure que je trouve des moyens pour me sortire cette situations » ou encore, dans une autre production »

(5) * «j'aimerais vous démander une année de conger afin que je puisse travailler pour obtenir un bon budgé pour étudier sans soucis ».

\section{Deuxième niveau : sur les arguments utilisés}

De façon générale, les arguments convoqués par les étudiants portent sur deux plans: leurs motivations, leurs besoins personnels et le bien collectif. Quand il s'agit de faire référence au collectif, l'argumentation est pragmatique: la décision est évaluée en fonction de ses conséquences favorables ou défavorables, de son utilité (Bellenger, 1996). Les étudiants évoquent la "nécessité du renforcement", le fait que le module soit « indispensable», «important», « essentiel», «primordial» dans le parcours des étudiants étrangers (6 copies). Trois arguments sont invoqués pour attester de la nécessité du programme : celui-ci apporte un approfondissement de la langue par le travail des pratiques contrastées, orales ou écrites, et de divers mécanismes langagiers (cités dans 5 copies); il permet d'apprendre la langue à un rythme moins stressant que celui du parcours B (2 copies); le parcours A donne la possibilité aux étudiants de poursuivre des études à l'université de Genève en vue de la complétion d'une maitrise ou d'un doctorat (2 copies). Les arguments qui abordent les conséquences négatives de la suppression développent trois aspects : l'ampleur des conséquences de la suppression, due au fait que beaucoup d'étudiants sont dans cette situation ( 1 copie) ; les choix de carrière des étudiants qui se voient contrecarrés ( 2 copies); la réduction du nombre d'étudiants voulant étudier le français (1 copie). Notons aussi, dans les arguments collectifs, la valorisation du français comme langue de prestige: les étudiants ont d'ailleurs recours à des définitions slogans : " cette belle langue de Molière ", « la langue de l'amour». À ceci s'ajoute l'argument voulant que l'unité de formation dispense $\mathrm{du} »$ matériel culturel suisse ».

Quand il s'agit d'arguments d'ordre personnel, les étudiants font référence à leurs besoins et à leurs motivations. Les illustrations font ici appel aux valeurs. Les étudiants n'hésitent pas à convoquer les sentiments pour étayer leur position: on veut culpabiliser le directeur en lui montrant les efforts investis pour arriver à suivre le parcours $\mathrm{A}$ :

(6) * «J'ai quitté mon pays et me suis installé à Genève avec le seul rêve dans ma tête ",

(7) * « Je suis une élève qui a travaillé pendant une année pour arriver à votre cours".

C'est la stratégie également employée par un des étudiants ayant interprété différemment la consigne :

(8) * « Je dois suivre ce cours parce que c'est ma rêve d'enfance d'étudier à

l'ECLF à Genève (...) J'ai battu jusqu'au bout ».

Certains lui promettent la gratitude :

(9) * « je vous serais extrêmement reconaissant si vous pourriez faire un petit effort financièrement ",

(10) * « on vous mercie si vous ne supprimez pas ce cours ». 
En somme, il apparait que les arguments invoqués sont sensiblement de même nature : on a recours aux faits, aux valeurs et aux sentiments. Les arguments sont évoqués dans le tout-venant, c'est la marque d'un premier jet. Mis à part une argumentation pragmatique par le raisonnement causal, dans lequel on énonce les conséquences de la suppression, il est difficile de saisir la stratégie argumentative déployée. Un travail supplémentaire de documentation sur la thématique de la controverse et une réorganisation cohérente de l'enchainement des arguments, en adoptant une stratégie pour convaincre le directeur, pourraient donner plus de force argumentative aux productions.

\section{Troisième niveau : sur les phénomènes de textualisation...}

Selon le Cadre Européen Commun de Référence pour les langues (Conseil de l'Europe, 2012), le locuteur de niveau C1 s'exprime «sur des sujets complexes de façon claire et bien structurée et manifeste son contrôle des outils d'organisation, d'articulation et de cohésion du discours». Du corpus de textes analysés, nous n'avons pas relevé de problèmes récurrents quant à l'usage des organisateurs textuels. Notons que ceux-ci se trouvent en nombre limité, sont peu variés, mais que leur emploi est pertinent au contexte de communication. Même constat pour les phénomènes de cohésion nominale, lesquels semblent bien maitrisés par les étudiants. Les difficultés les plus fréquentes se rapportent à la cohésion temporelle et à l'usage des temps du verbe ainsi qu'à la construction des formules de salutations finales.

Sur les verbes, le type de discours de la lettre de demande implique un monde conjoint au monde ordinaire de l'argumentateur. Les paramètres de l'acte de production (la durée de l'acte de production, par exemple) constituent donc un critère pertinent pour l'analyse des mécanismes de cohésion verbale. Dans le cours du processus expositif-interactif, c'est la gestion des axes de référence temporelle distincts de la durée de l'acte de production qui pose problème aux élèves. On ne s'étonnera pas de ce résultat: la diversité de paramètres de contrôle possibles, les fonctions de temporalité première présentes, dans le discours interactif, donnent à celui-ci une complexité supérieure aux autres types de discours (Bronckart, 1997).

Dans les productions recensées, le repérage des procès s'effectue par rapport à la temporalité de l'acte de production. Les étudiants utilisent donc tous l'indicatif présent comme temps de base. Le procès de «la réception de la décision de la suppression du parcours A » est mis en rapport avec d'autres éléments. Et c'est dans cette mise en rapport du procès avec d'autres évènements, de durée variable, antérieurs, simultanés ou postérieurs que se situent les principaux obstacles.

Dans l'usage du conditionnel :

(11) « je vous souhaiterais de revenir sur cette décision»

27 Les erreurs surviennent au moment de la construction de phrases complexes qui demandent le respect des règles de concordance des temps (5 copies) :

(12) * « je me propose comme bénévole à l'UNIGE à fin de reunir les fonds monétaires qui puisse aider à ouvrir ce cours et qui peut bénéficier à tous les élèves ",

(13) * «Hier soir, j'ai reçu la nouvelle que vous avez décidé de suprimer le cours DE1 à cause des raisons budgétaires ", 
(14) * «J'ai entendu que le cours du renforcement linguistique est supprimé pour des raisons budgétaires ",

$(15) *$ « et je vous serai vraiment reconaissant si vous pourriez faire un petit effort ».

Des erreurs surviennent en lien au marquage de l'antériorité dans la contextualisation de la demande :

(16) * «J'ai choisi ce cours la semaine prochaine »,

(17) * «j'étais tellement déçue de savoir que le parcours A, avec le renforcement linguistique, était supprimé ».

Parfois c'est la formulation d'un souhait qui pose problème :

$(18)$ * «j'espère que vous avez changé d'avis après avoir lu cette pétition »,

(19) * «j'espère que vous pourriez trouver la solution de ce problème budgétaire ",

(20) * «J'espère qu'il y aura des autres étudiantes qui vous ecrirais des lettres comme la mienne ».

Des problèmes surviennent enfin dans l'énoncé de conséquences futures :

(21) * «Vous devriez bien réfléchir cette décision car il y a beaucoup d'étudiants qui ne peuvent plus continuer leurs études ou leurs carrières et la probabilité de trouver un bon travail doit être très difficile ",

(22) * «C'est vrai que ma situation budgétaire est difficile pour instant. Mais je vous jure que je trouve des moyens pour se sortire cette situations ».

Les autres difficultés relèvent de constructions syntaxiques erronées :

(25) * «Je vous remercie en avance, pris d'agréer monsieur »,

(26) * « En attendant votre réponse, je vous prier d'agréer, Mes meilleures salutations.

Parmi celles-ci, relevons la place et l'accord de l'adjectif meilleur et du nom salutations :

(27) * «Avec mes salutations de meuilleurs ».

Souvent, les difficultés d'ordre syntaxique s'entremêlent à des difficultés d'ordre sémantique :

(28) * «Dans l'attente de votre décision, que j'espère favorable, je vous prie de croire, Monsieur, mes sentiments respectueux ».

Ici, c'est la valence du verbe croire qui pose problème, mais aussi la jonction du croire et de sentiments respectueux, sans le passage par en l'expression de.

Pour se faire une représentation complète et contrastée des obstacles des élèves, nous analyserons maintenant le corpus de jeunes élèves produisant un genre argumentatif en français langue de scolarisation. 


\section{Analyse du corpus d'élèves de l'école primaire}

\section{Premier niveau : sur la situation de communication}

La consigne de production du corpus «Valais 6e primaire » apparait sur le support de cours où les élèves doivent rédiger leur RCL. Plus exactement, la lettre source constitue elle-même la consigne de l'activité. Un courrier fictif doit en effet servir à déclencher la production d'une RCL :

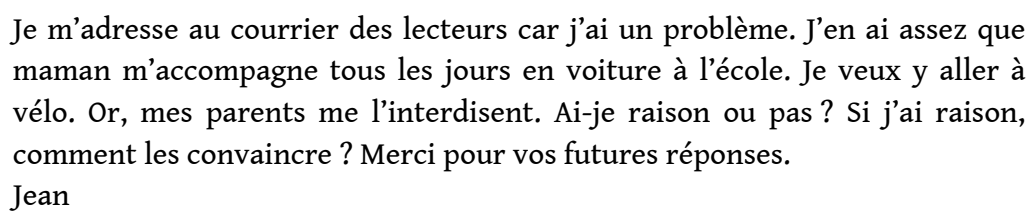
maman m'accompagne tous les jours en voiture à l'école. Je veux y aller à vélo. Or, mes parents me l'interdisent. Ai-je raison ou pas? Si j'ai raison, comment les convaincre? Merci pour vos futures réponses. Jean

33 La pratique sociale de référence de la RCL se trouve transposée dans un contexte scolaire, et transformée en activité servant l'apprentissage. L'activité scolaire de production initiale consiste ainsi à répondre à un personnage (Jean) qui s'est lui-même adressé à la rubrique RCL d'une publication fictive pour enfants. La situation de communication impliquée par l'activité scolaire est d'une certaine complexité: les élèves doivent s'adresser à un personnage au sujet duquel ils savent relativement peu de choses, mais doivent encore lui donner tort ou raison et le cas échéant l'aider à convaincre ses parents.

A en juger par les 16 productions du corpus, les élèves saisissent tous qu'il leur est demandé de s'adresser à Jean au sujet du déplacement en vélo à l'école. Deux phénomènes récurrents parmi les textes des élèves et relatifs à la représentation de la situation de communication appellent cependant nos commentaires: la construction de situations hypothétiques et l'auto-centration.

\section{Construction de situations hypothétiques}

Dans 11 productions sur 16, les élèves s'emploient à concevoir des situations hypothétiques, comme pour étoffer les informations relativement sommaires apparaissant dans la lettre source. De nombreux élèves imaginent, par exemple, des situations où l'utilisation du vélo serait problématique (nous soulignons) :

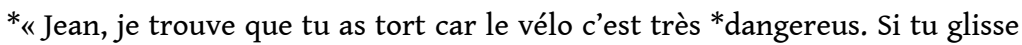
sur une flaque d'eau et tu ouvre le genou ou tomber et t'ouvrir le crane et qu'il n'y a personne tu va à l'hôpital Alors va à l'école en voiture »

(2) * "Jean tu a tort le vélo ses bien Mais tu peut tomber, avoir des accidents très grave. Mais ces trop dangereux on peut de voler le vélo si il ni a pas le cadenas. Je crois que tes parents ont raison, alors laisse ta maman où ton papa $\mathrm{t}$ amené $\mathrm{A}$ l'école en voiture c'est moins dangereux et tes plus en sécurité. »

(3) * « Je suis d'accor avec tes parents parce si on fait du vélo on peut tonber sur la route et c'est dangereu et il faut aitre écipée pour faire du vélo et pour aller à l'école il faut avoir tout le matérielle »
}

Dans les trois exemples précités, l'imagination par les élèves de situations hypothétiques leur permet d'inscrire leurs prises de position dans un arrière-plan plus précis, et donc de réfléchir à partir d'une situation concrète (bien que fictive). En d'autres cas, l'imagination 
par les jeunes scripteurs de situations fictives prend une proportion plus importante encore (nous soulignons) :

(4) * «Jean, ta maman a résont parce que si tu te fait voler le vélo ou tu na pas la vinniet et tu glise tu réye la voiture tu dois péyier les réparation de la voiture»

(5) * «Jean tu as tore tu pour est faire du vélo avec tes parents comme sa il ne sincètera pas pour toi. est si tu fairais du vélo pour aller a l'école est qu'il pleu comment tu ferais? C'est petettre se que tu pourais fair. »

Ici, après avoir pris position, les auteurs tendent à produire une argumentation par hypothèses qui revient à «mobiliser les conséquences possibles d'un fait ou d'une opinion ou d'un choix et de les utiliser comme appât ou comme menace » (Bellenger, 1996, p. 61). S'il s'agit d'une authentique conduite argumentative, il n'en demeure pas moins qu'elle passe par une forme de restriction du contexte de réflexion à des situations bien précises. En ce sens, les élèves s'appuient donc sur des considérations relativement peu généralisables. Il arrive enfin qu'un élève réponde à Jean en évoquant uniquement une suite de situations hypothétiques sans explicitement prendre position :

(6) ${ }^{*}$ «Si tu mets un casquet sa sera moin dangereux. Met si tu ne sais pas faire du vélo ne vas pas à l'école à vélo. Si ta beaucoup de chose ne prend pas le vélo et si il fait movée temps fait attonsiont."

Dans ce cas, l'élève tend à généraliser son expérience heureuse du déplacement en transports publics en y incluant le destinataire de sa lettre (le changement pronominal entre «J'aime bien prendre le car » et «Tu es avec tes copains » est de ce point de vue éloquent). Le jeu d'identification entre cet élève et Jean parait même aller plus loin : c'est ce que donnerait à voir l'enchainement des deux dernières propositions de cette réponse

(7) * « Cher Jean,

Si ton école est loin de ta maison va à l'école avec ta mère. Moi je dois prendre le car. J'aime bien prendre le car. Tu ais avec tes copains. Moi mon école est trop loinpt pour aller en vélo. Alors continue à allé avec ta mere " situations possibles. C'est en tout cas ce qui semble retenir son attention, signe de la difficulté de la tâche qui lui est demandée.

étranger aux phénomènes de production d'hypothèses : c'est par un travail d'inférences important que passent nombre d'élèves pour pouvoir rédiger leur RCL. Autrement dit, l'imagination de situations hypothétiques peut être une manière, pour les élèves, de dresser le décor de leurs réflexions, mais aussi de produire certains types d'arguments contextuellement restreints. Ce besoin pourrait d'ailleurs expliquer le deuxième phénomène récurrent que nous annoncions : l'auto-centration.

\section{Auto-centration}

Dans 7 productions sur 16, les élèves partent de leurs propres expériences pour donner de la consistance à leurs lettres. Il arrive que l'auto-centration (nous soulignons) serve à la fois la construction d'une situation hypothétique et, indirectement, l'argumentation : 
(«Moi, mon école est trop loin pour aller en vélo. Alors continue à aller avec ta mère »). En d'autres termes, il semblerait que cet élève hisse son expérience des déplacements à l'école - ainsi que sa propre situation géographique - sinon au rang d'argument, au moins à celui de conditions partagées par Jean et lui-même. Un nouvel exemple de ce phénomène d'auto-centration étendue au destinataire nous est donné par une autre production:

(8) * «Jean ta tort, parce que les montées c'est très dure, à moin que tu es sportif. Moi je n'aime pas les montées »

En l'occurrence, l'auteur de cette réponse - qui vit dans un village alpin - semble s'adresser à un Jean qui habiterait lui aussi à flanc de montagne: si l'auto-centration produit ici un argument valable dans le contexte convoqué, il ne tient pas davantage compte des inconnues entourant Jean que ne le faisait la production précédente. La valeur de tels arguments est elle-aussi contextuellement restreinte, et il apparait que ces deux élèves demeurent à un niveau peu élevé de généralisation. Même lorsqu'un élève parvient à développer des arguments à valeur générale, l'auto-centration peut s'ajouter à la production, un peu à la manière d'une pièce rapportée (nous soulignons) :

(9) * « Jean, tu as tort car le vélo s'est dangereux, il y a de plus en plus de mort et s'est fatiguant et du doit être ordre moi j'aime bien le vtt et le vélo car j'aime le sport.»

Le scripteur conclut sa lettre en faisant part de son gout pour le vélo, alors même qu'il vise à dissuader Jean d'en employer un pour se rendre à l'école. L'auto-centration semble ainsi polariser l'attention de l'élève, quand bien même il se montre capable d'énoncer des arguments à valeur générale $(*$ (...) le vélo c’est dangereux, il y a de plus en plus de morts et c'est fatiguant (...)»)

Un certain déséquilibre se donne donc à voir à travers ces dernières productions initiales: l'auto-centration y occupe une place plus importante que les contenus généralisables à toutes les situations. Tout comme pour les phénomènes de construction de situations hypothétiques observés, ceux d'autocentration pourraient être appréhendés comme des stratégies mises en place par certains élèves pour pallier le caractère parcellaire des informations fournies par la lettre source. Alors que, dans le premier cas, ce sont des situations fictives qui sont imaginées, l'autocentration convoque pour sa part l'expérience personnelle des scripteurs - quitte à la substituer parfois à l'identité du destinataire.

\section{Deuxième niveau : sur les arguments utilisés et leur place dans la planification du texte}

Les 16 productions de notre corpus permettent de constater diverses conduites argumentatives. Comme nous l'avons esquissé, certains élèves recourent à l'émission d'hypothèses pour préciser l'arrière-plan référentiel de leur argumentation, tandis que d'autres prêtent apparemment une valeur d'argument à des situations hypothétiques qu'ils ont imaginées (ou se limitent à en produire), mais auxquelles nous serions enclins à n'accorder qu'une valeur d'exemples.

De la même manière, si la référence à des expériences personnelles peut parfois être bienvenue (au fond, à travers la RCL, il est aussi demandé aux élèves de socialiser avec ce 
Jean qui a probablement un âge approchant du leur, mais au sujet duquel ils ne savent pas grand-chose) et même aider le destinataire de leur lettre à convaincre ou persuader ses parents, d'autres productions laissent à penser que certains élèves entendent convaincre Jean de renoncer à son envie d'aller à l'école à vélo en se référent uniquement à leurs propres expériences négatives des deux-roues.

À travers les quelques arguments apparaissant au fil de ces productions, on observe 8 élèves sur 16 se montrer particulièrement sensibles à la dangerosité de ce moyen de transport («car le vélo c'est très dangereux »; « (...) ne pas aller à l'école avec le vélo quand tu peux faire beaucoup d'accidents » : « tu peux te faire très mal en vélo ! »...). Sur l'ensemble des productions, une seule donne raison à Jean, et propose un argument écologique (" Aller à vélo est bien on pollue pas »). D'autres productions s'attèlent enfin à donner des conseils à Jean, sans pour autant lui offrir d'arguments (nous soulignons) :

(10) ${ }$ « Cher Jean,

Moi, je vais à l'école en trotinette. Au début, j'ai demander a mes parents si je pouvais et ils étaient d'accord. Mais moi, pour toi, j'ai plusieurs conseils. En voici 2: demande le plus gentiment possible. Le 2e est: fais des choses pour tes parents comme aller au magasin. Suis bien mes conseils et hop ! ça marche!!! Merci de m'écouter!!! »

Qu'elles se focalisent sur des conseils pour aider Jean, sur l'imagination d'hypothèses ou qu'elles se développent d'un point de vue autocentré, la majorité de ces productions initiales fait apparaitre des stratégies argumentatives attestées, mais contextuellement restreintes. Cet état de fait met ainsi en évidence la grande dépendance, dans cette activité, entre la représentation de la situation de communication spécifique impliquée par la consigne et le développement des arguments.

\section{Troisième niveau : sur des phénomènes de textualisation}

Notre analyse a montré que les élèves du corpus "Valais 6e primaire " s'appuient fréquemment sur la construction de situations hypothétiques pour diriger leurs conduites argumentatives. Ce n'est donc pas une surprise de constater, dans ces mêmes productions, un phénomène digne d'intérêt relatif à la textualisation des hypothèses. Les copies donnent en effet à voir que les élèves recourent à deux types de moyens langagiers pour signifier la possibilité, la condition ou l'hypothèse.

Parmi les moyens langagiers employés à cet effet par les élèves, nous retrouvons ainsi majoritairement les enchainements « Si+indicatif » (6 copies sur les 11 qui font apparaitre des situations hypothétiques), alors que deux copies seulement présentent une tentative d'utilisation du conditionnel, ou de l'enchainement « Si+imparfait+conditionnel » :

$(11) *_{«}(. .$.$) tu pour est faire du vélo avec tes parents comme sa il ne sincètera$ pas pour toi. est si tu fairais du vélo pour aller a l'école est qu'il pleu comment tu ferais ? C'est petettre se que tu pourais fair. "

(12) * « Essaie de demmander à tes parents en les rassurants que tu mettrais un casque.»

51 Dans ce cas, nous pouvons d'ailleurs relever que les élèves emploient avec le conditionnel un mode qu'ils ne sont pas encore supposés maitriser à leur âge (le conditionnel est simplement introduit en 6e primaire). Cette observation peut avoir un certain intérêt quant à la planification des contenus d'enseignement pour la 6e primaire: il serait 
pertinent de chercher à renforcer réciproquement l'apprentissage du conditionnel et celui de la RCL.

Les élèves font également fréquemment recours, dans 5 copies sur les 11 retenues, à des verbes modalisateurs exprimant la possibilité (« Mais tu peux tomber, avoir des accidents très graves (...) on peut te voler le vélo si il n'y a pas le cadenas »). Là encore, une séquence d'enseignement portant sur la RCL serait l'occasion de renforcer les apprentissages portant sur ces constructions verbales.

\section{En guise de conclusion}

\section{Analyse contrastive des deux corpus}

La comparaison des deux corpus met en évidence la pertinence de la démarche générale proposée d'analyse descendante en trois niveaux. La saisie des obstacles relatifs à l'adaptation à la situation de communication, abordés parfois de manière trop rapide par les enseignants, s'avère par ailleurs déterminante pour comprendre les difficultés des élèves aux deux autres niveaux: cette adaptation détermine le choix des arguments mobilisés, la planification du texte et même le choix de procédures de textualisation. Comme nous l'avons évoqué dans le premier corpus, il s'agit des problèmes de prise en charge énonciative et d'utilisation des temps verbaux en rapport avec la fictionnalisation de la situation de communication proposée par la consigne. Quant au deuxième corpus, on voit apparaitre le rapport très étroit entre le processus de fictionnalisation des paramètres de la situation de communication et l'alternance entre la construction de situations hypothétiques, ainsi que les obstacles observables dans l'utilisation du si + indicatif.

Le contraste entre les deux genres montre également l'importance de la cohérence générique spécifique à ces deux formes de la correspondance argumentative qui se distinguent aussi bien par la nature du destinataire, celle du but à atteindre et par leurs lieux sociaux de production. La majorité des adultes d'origine non francophone connaissent bien ces aspects particuliers du genre et, même s'ils sont en train d'apprendre une nouvelle langue - le français -, ces étudiants transfèrent dans leurs productions leurs connaissances $d u$ contexte ou celles apprises dans leur langue première. La connaissance des situations semble également nécessaire pour bien articuler l'usage des prises en charge énonciatives (pronoms) et de la gestion des temps du verbe. En ce qui concerne les élèves de l'école primaire, les connaissances relatives à la situation de communication et à l'espace de contraintes générales du genre « réponse au courrier des lecteurs » semblent indispensables pour développer la cohérence générique du texte.

Les supports proposés aux apprenants se révèlent fondamentaux pour interpréter la situation de communication, ils créent un espace de travail et d'interaction. Ils fixent les contraintes du genre et les relations d'intertextualité, ces dernières pouvant se manifester comme réponse à un autre texte écrit ou à des propos tenus. Dans un cas, l'exemplaire de texte auquel l'élève doit répondre fournit des exemples sur le texte à produire. Dans l'autre, l'absence d'exemple de texte oblige l'élève à tout reconstruire luimême.

Les consignes particulières des deux situations sont par ailleurs déterminantes pour comprendre le choix et le développement des arguments proposés. Les enjeux 
argumentatifs des deux situations sont différents, et la compréhension de ces enjeux oblige à une analyse spécifique des arguments pertinents en fonction du but escompté par le texte. Les arguments produits dépendent en outre de la mobilisation de savoirs disponibles chez les apprenants (savoirs qui s'avèrent très différents entre les adultes et les élèves de l'école primaire, ou en fonction de la formation et de la culture d'origine des étudiants de français langue étrangère). Aucun des contextes de production n'introduit de facilitateurs, que ce soit dans les arguments à mobiliser ou dans le modèle du texte à produire.

Dans les deux cas, la prise en considération du dédoublement du genre (social et scolaire) et des opérations de fictionnalisation permet une meilleure compréhension des erreurs observées et des obstacles qui sont à la source des apprentissages. Le réalisme et la proximité de la situation de communication scolaire proposée (la lettre au directeur de l'institution) contribuent à faire générer facilement des arguments. Si certains apprenants confondent quelque peu l'enjeu fictif de celle-ci (on mêle la suppression du parcours avec son renvoi personnel), la plupart se prêtent au jeu avec aisance, en témoignent la gestion des voix énonciatives (la leur et celle des autres apprenants allophones dans la même situation) et la formulation explicite d'une demande. Dans le cas du corpus "Valais 6e primaire ", l'identité fictionnelle du Jean auquel doivent s'adresser les élèves est un effet direct du dédoublement générique impliqué par la scolarisation de la RCL. En effet, le genre RCL vaut dans ce contexte scolaire tout autant comme un genre pour communiquer que comme un genre pour apprendre. Il s'ensuit que les moyens d'enseignement convoquent une situation fictive pour placer les élèves en situation de production: ces derniers doivent faire comme si Jean existait, tout en sachant qu'il n'est que fiction. Nous avons montré ailleurs (Vuillet, Mabillard, Tobola-Couchepin, \& Dolz, sous presse) que la représentation des paramètres de la situation de communication revêt une importance capitale pour la production d'un genre et que cette représentation comprend également la prise en considération du contexte de production spécifiquement scolaire. L'observation et l'analyse des traces de la construction par les apprenants des paramètres de la situation de communication conditionnent la compréhension de certaines procédures aussi bien argumentatives (en témoigne la nature des arguments produits) que linguistiques. Autrement dit, la seule prise en considération du profil spécifique des élèves ou des étudiants nous apparait insuffisante pour traiter leurs difficultés. Il s'agit également de se doter d'outils d'analyse qui prennent en compte l'ensemble des données de la situation scolaire de production d'un exemplaire de genre: la consigne scolaire et les paramètres de la situation de communication sont à ce titre du plus grand intérêt.

\section{Les dispositifs didactiques pour dépasser les principaux obstacles observés}

En termes de dispositifs didactiques, les résultats de l'analyse contrastée des erreurs et des capacités des apprenants des deux corpus nous ont permis de dégager les principaux obstacles et les zones de développement. Les difficultés les plus fréquentes dans le corpus «ELCF » se rapportent aux mécanismes de textualisation et, principalement, à l'harmonisation des temps du verbe et à l'utilisation des formules de salutations finales. Nous sommes en présence de locuteurs adultes, connaissant des normes du genre, il n'est donc pas surprenant que les principales difficultés se lient à la mise en mots du texte, que 
ce soit sur le plan de la mise en rapport des événements dans le texte ou dans la formulation d'un appel à poursuivre le dialogue ou de salutations finales. Du côté des élèves de 6e primaire, un obstacle important réside dans les difficultés rencontrées par les élèves lorsqu'il s'agit pour eux d'écrire à une personne inconnue. Ces difficultés appellent ainsi l'aménagement anticipé d'activités scolaires portant sur la représentation de la situation de communication, ainsi que le développement d'autres activités portant sur des procédures linguistiques utiles à l'expression de relations d'hypothèses.

Il ne s'agit pas simplement d'établir une typologie des erreurs lexicales, grammaticales ou textuelles les plus fréquentes pour chaque genre, mais de saisir l'origine des obstacles rencontrés. Ces obstacles, comme nous l'avons montré, ont une nature complexe par le fait que la généricité suppose un espace de contraintes particulières orientées par les situations d'interaction. La cohérence générique et la cohésion du texte obligent l'évaluateur à disposer d'informations précises sur les consignes proposées et à prélever des indicateurs en fonction des trois niveaux d'analyse, mais aussi en les croisant. Pour dépasser les annotations intuitives sur le texte ou l'évaluation par une liste de critères du genre juxtaposés, nous proposons une meilleure connaissance du genre et un travail de recherche préalable sur les obstacles à l'apprentissage. Une fois mieux connus les erreurs et les obstacles à la source des problèmes d'apprentissage, il est possible d'améliorer l'évaluation ciblant davantage les critères sur la cohérence générique et sur les besoins des apprenants.

60 Une simple catégorisation des erreurs orthographiques ou syntaxiques n'est pas suffisante pour rendre compte des capacités des apprenants en matière d'écriture. Seules des situations de communication et des tâches complexes d'écriture autonome permettent d'évaluer l'ensemble de capacités mobilisées. Ces tâches ne peuvent pas être les mêmes pour des adultes non francophones que pour des jeunes élèves ayant le français comme langue de scolarisation mais dans les deux cas l'enseignant a besoin d'une modélisation didactique du genre enseigné pour évaluer les productions et évaluer les apprentissages. De ce point de vue, les résultats des analyses réalisées dans cette recherche fournissent aux enseignants des outils pour évaluer et comparer d'une manière large et ciblée les performances de leurs élèves. La catégorisation des obstacles proposée en fonction des opérations de production textuelle et des mécanismes d'apprentissage permet de mieux articuler les choix didactiques aux performances des apprenants. Il est vrai que les obstacles lexicaux, grammaticaux et orthographiques peuvent faire l'objet d'interventions, de corrections et de régulations locales pertinentes (Surian, 2015) mais une vision globale des différentes dimensions impliquées dans l'écriture permet une explicitation et hiérarchisation des obstacles à dépasser et une planification des articulations à prendre en considération. Les interventions des enseignants peuvent alors s'organiser en fonction de dispositifs plus complets, dans des lieux de régulation successifs et différenciés.

61 Dans les deux cas et pour les deux populations, l'élaboration de dispositifs d'enseignement pour écrire des textes argumentatifs exige d'une part une réflexion sur l'influence des tâches d'écriture et la confection d'activités scolaires sur les situations de communication qui prennent davantage en considération les phénomènes de fictionnalisation. Concernant la recherche d'arguments et la planification argumentative, les besoins et les obstacles constatés sont relativement différents chez les adultes non francophones et pour les élèves en difficulté de l'école primaire mais, dans les deux cas, un travail pour mieux étayer avec des arguments les opinions en développant des 
stratégies argumentatives plus complexes reste important. Au niveau de la textualisation, les dysfonctionnements sont assez différents, l'évaluation des obstacles permet alors de repérer les mécanismes de cohésion et de connexion textuelle qui méritent un travail collectif à côté des annotations particulières ciblant un seul texte mais qui peut être un levier pour l'ensemble des apprenants. En ce qui concerne les difficultés lexicales, syntaxiques et orthographiques, nous constatons un nombre important d'obstacles divers et différenciés pour les deux populations. De nouveau, le choix peut dépasser les corrections et les annotations locales dans les copies dans des régulations strictement individuelles pour permettre un travail d'explicitation collectif du fonctionnement de la langue française. Le texte devient alors un prétexte pour travailler sur la langue mais cherchant une intégration co-textualisée. Enfin, étant donné l'impact du choix des supports dans la mise en place de situations de production, il s'avère nécessaire de réfléchir à leur place et à leur rôle à l'intérieur du dispositif d'enseignement du genre textuel.

Nous pouvons donc conclure que, même si les obstacles d'écriture des apprenants de français langue étrangère sont assez différents de ceux des jeunes élèves qui ont le français comme langue de scolarisation, l'évaluation initiale des capacités dans des tâches complexes relatives à des genres textuels reste une condition pour adapter les choix didactiques des enseignants aux besoins des apprenants.

\section{BIBLIOGRAPHIE}

Bellenger, L. (1996). L'argumentation; Des techniques pour convaincre. Paris : ESF.

Bernié, J.-P. (1998). «Fondements théoriques d'une didactique interactionniste de la langue maternelle ». In Brossard, M. \& Fijalkow, J. (Éd.), Apprendre à l'école : perspectives piagétiennes et vygotskiennes. Bordeaux : Presses universitaires. pp. 155-197.

Bronckart, J.-P. (1997). Activité langagière, textes et discours. Pour un interactionnisme sociodiscursif.Neuchâtel et Paris : Delachaux et Niestlé.

Conseil de l'Europe, Cadre européen des langues, page consultée le 30 mars 2012, http:// www.coe.int/t/dg4/linguistic/Source/Framework_FR.pdf.

de Pietro J.-F., Erard, S. Kaneman, M. (1997). « Un modèle didactique du débat : de l'objet social à la pratique scolaire ». Enjeux, 39/40, 100-129.

Dolz, J. \& Abouzaid, M. (2015). «Pluralité des genres et singularité du texte : tensions constitutives de la didactique des langues ». Forum Lecture, 2. Consulté le 14.09.2015, http:// www.leseforum.ch/myUploadData/files/2015_2_Dolz_Abouzaid.pdf.

Dolz, J. Gagnon, R. et Vuillet, Y. (2011). Production écrite et difficultés d'apprentissage. Genève, Carnets de la Section des Sciences de l'Education.

Dolz, J., Mabillard, J.-P., Tobola-Couchepin, C. \& Vuillet,Y. (2009). Didactique de l'expression écrite et difficultés d'apprentissage, une étude exploratoire sur l'enseignement d'un genre textuel argumentatif à l'école primaire. Revue suisse des sciences de l'éducation. vol. 33, n 3. pp. 541-564. 
Dolz, J. \& Schneuwly, B. (1998). « Genres et progression en expression écrite : éléments de reflexion à propos d'une expérience romande ». Enjeux, 37/38, 49-75-

Dolz, J., Noverraz, M. \& Schneuwly, B., (2001). S'exprimer en français. Séquences didactiques pour l'oral et pour l'écrit. Bruxelles, De Boek.

Schneuwly, B. \& Dolz, J. (1997). « Les genres scolaires. Des pratiques scolaires aux objets d'enseignement ». Repères, $\mathrm{n}^{\circ}$ 15. pp. 27-40.

Schneuwly, B. (1997). Parole et fictionnalisation. Une voie pour l'enseignement de l'oral. Éléments pour une conférence donnée à la PUC-LAEL, São Paulo, Brésil.

Surian, M. (2015). Enseigner la production orale et écrite en classes d'accueil. Thèse 617 de la Faculté de psychologie et des sciences de l'éducation. Genève : Université de Genève.

Vuillet,Y., Mabillard, J.-P., Tobola-Couchepin, C. \& Dolz, J., (sous presse). Dédoublement du genre et des opérations de fictionnalisation, comment faire « comme si c'était vrai »? Education et didactique, Rennes : PUR.

Vygotski, L. S. (1934/1997). Pensée et langage. Paris : La Dispute.

\section{NOTES}

1. Les données utilisées dans cette contribution proviennent de deux recherches : la première (fond DORE 13DPD3-120167), plus vaste, a été menée par J. Dolz, J.-P. Mabillard, Catherine TobolaCouchepin \& Y. Vuillet; la seconde (FNRS- IZK0Z1_139572/1) est liée au stage de recherche postdoctoral de R. Gagnon.

\section{RÉSUMÉS}

Deux genres argumentatifs : la "lettre de demande " et la "réponse au courrier des lecteurs" ont été modélisés didactiquement. L'analyse contrastive de textes argumentatifs rédigés par des étudiants adultes en français langue étrangère et par des élèves de l'école primaire a permis une catégorisation des erreurs caractéristiques et des capacités langagières en cours de développement. Des hypothèses sur les zones de proche développement permettent aux auteurs de suggérer l'élaboration de nouveaux dispositifs didactiques pour dépasser les obstacles observés.

Two argumentative genres "request letter" and "response to readers' letters" were modeled didactically for teaching. The contrastive analysis of the argumentative texts produced by adult students in French as a foreign language and by students from the primary school allowed a categorization of the characteristic errors and the language capacities that are being developed for these two categories of learners. The hypotheses on the zones of proximal development allow the authors to suggest the elaboration of new didactic instruments to overcome the obstacles observed. 
INDEX

Mots-clés : dispositif didactique, capacités langagières, erreurs, genres argumentatifs, obstacles d'apprentissage, production écrite, zone de proche développement

Keywords : didactic instruments, language capacities, argumentative genres, learning obstacles, written production, zones of proximal development

\section{AUTEURS}

JOAQUIM DOLZ

ROXANE GAGNON

YANN VUILLET 